Recepción: 20 / 12 / 2018

Aceptación: 28 / $01 / 2019$

Publicación: 20 / 03 / 2019

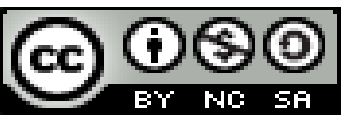

Ciencias de la salud

Artículo de revisión

\title{
Análisis de la actividad física y su importancia en el sistema educativo ecuatoriano: carrera Gestión Emresarial de la Universidad Estatal de Sur de Manabí \\ Analysis of physical activity and its importance in the Ecuadorian educational system: emresarial management career at the State University of Sur de Manabí
}

Análise da atividade física e sua importância no sistema educacional equatoriano: carreira de gestão emissária na Universidade Estadual de Sur de Manabí

Juan M. González-Morán ${ }^{\mathrm{I}}$ marlon.gonzález@unesum.edu.ec

Marcos G. Cañarte-Castillo II marcos.cañarte@unesum.edu.ec

Rubén D. Acebo-Calderón ${ }^{\text {III }}$ ruben.acebo@unesum.edu.ec

Regalado F. Katty-Glenda IV katty.regalado@unesum.edu.ec

Carlos A. Zea-Barahona V carlos.zea@unesum.edu.ec

Xavier E. Soledispa-Rodríguez VI xavier.soledispa@unesum.edu.ec

Correspondencia: marlon.gonzález@unesum.edu.ec

Magister Docencia Universitaria e Investigación Educativa, Licenciado en Educación Física, Docente Ocasional de la Universidad Estatal del Sur de Manabí, Manabí, Ecuador.

Magister Docencia Universitaria e Investigación Educativa, Licenciado en Educación Física y Deportes, Docente Ocasional de la Universidad Estatal del Sur de Manabí, Manabí, Ecuador.

Diplomado en Autoevaluación y Acreditación Universitaria, Licenciado en Ciencias de la Educación Especialidad Cultura Física y Deportes Docente Encargado del Área de Deportes de la Universidad Estatal del Sur de Manabí, Manabí, Ecuador.

Magister Docencia Universitaria e Investigación Educativa, Licenciada, Administrativa Departamento de Bienestar Estudiantil de la Universidad Estatal del Sur de Manabí, Manabí, Ecuador.

Magister Docencia Universitaria e Investigación Educativa, Economista, Docente Titular Principal Tiempo Completo de la Universidad Estatal del Sur de Manabí, Manabí, Ecuador.

Especialista en Consultoría de Proyectos, Economista, Docente Titular Principal Tiempo Completo de la Universidad Estatal del Sur de Manabí, Manabí, Ecuador. 
Análisis de la actividad física y su importancia en el sistema educativo ecuatoriano: carrera Gestión Emresarial de la Universidad Estatal de Sur de Manabí

\section{Resumen}

El desarrollo del presente artículo tiene como propósito analizar la actividad física y su importancia en el sistema educativo ecuatoriano: carrera gestión empresarial de la universidad estatal del Sur de Manabí, para la misma fue necesario incorporar la revisión de diferentes documentos, leyes y material electrónico con el fin de dar la respectiva organización a las ideas para plasmar valoraciones significativas en cuanto al tema. Es importante destacar, que la misma necesidad de transformar el sistema educativo, obliga al Estado ecuatoriano a introducir políticas educativas caracterizadas por generar cambios en sus mallas curriculares, es decir, se fija la actividad física como una posible estrategia fundamental para lograr resultados significativos en la dinámica pedagógica; pues, los estudiantes al realizar actividades físicas construye competencias personales, sociales, psicológicas, motoras que le ayudan a cumplir con los objetivos propuestos en cada nivel escolar, pues, su aprendizaje está determinado por un manejo eficiente de sus potencialidades desde una perspectiva integral. Sin embargo, cuando la acción didáctica se aleja de estos preceptos agrega un desmejoramiento en la calidad educativa, es allí, donde la Ley de Educación y Cultura (1983) en su artículo 2 numeral 1 - 2 que "es un deber primordial del Estado a que todos los Ecuatorianos tengan una educación integral y la obligación de participar activamente en el proceso educativo nacional". (p.1) Por ello, los estudiantes de la carrera gestión empresarial, poseen oportunidades de emplear su desarrollo físico, motor, social, personal durante sus actividades académicas, redimensionan su condición humana y construyen las bases para adecuar sus tareas profesionales en relación a las responsabilidades laborales como gerente o administrador que se anteponen en su desempeño; al ser preparados bajo una actividad física, se desarrollan como individuos dinámicos con posibilidades para descubrir y cultivar las cualidades que van a formarlo integralmente.

Palabras claves: Actividad Física; Sistema Educativo Ecuatoriano; Carrera Gestión Empresarial; Universidad Estatal del Sur Manabí.

\section{Abstract}

The purpose of this article is to analyze physical activity and its importance in the Ecuadorian educational system: business management career at the state university of the South of Manabí, for it was necessary to incorporate the review of different documents, laws and electronic material with the purpose of giving the respective organization to the ideas to capture significant valuations regarding the subject. It is important to emphasize that the same need to transform the educational 
system, forces the Ecuadorian State to introduce educational policies characterized by generating changes in their curricular meshes, that is, physical activity is fixed as a possible fundamental strategy to achieve significant results in the pedagogical dynamics; Therefore, when students carry out physical activities, they build personal, social, psychological, and motor skills that help them to achieve the objectives proposed at each school level, since their learning is determined by an efficient management of their potential from an integral perspective. However, when the didactic action moves away from these precepts it adds a deterioration in the educational quality, it is there, where the Law of Education and Culture (1983) in its article 2 numeral 1 - 2 that "it is a primary duty of the State to that all Ecuadorians have a comprehensive education and the obligation to participate actively in the national educational process." (p.1) Therefore, the students of the business management career, have opportunities to use their physical, motor, social, personal development during their academic activities, resize their human condition and build the bases to adapt their professional tasks in relation to the job responsibilities as manager or administrator that precede their performance; Being prepared under a physical activity, they develop as dynamic individuals with possibilities to discover and cultivate the qualities that are going to form it integrally.

Keys words: Physical activity; Ecuadorian Educational System; Business Management Career; State University of the South Manabí.

\section{Resumo.}

The purpose of this article is to analyze the physical activity and its importance in the Ecuadorian educational system: business management career at the state university of the South of Manabí, for it was necessary to incorporate the review of different documents, laws and electronic material with the purpose of giving the respective organization to the ideas to capture significant valuations regarding the subject. It is important to emphasize that the same need to transform the educational system, forces the Ecuadorian State to introduce educational policies, characterized by the changes in their curricular meshes, that is, physical activity is fixed as a possible fundamental strategy to achieve significant results in the pedagogical dynamics; Therefore, when students carry out physical activities, they build personal, social, psychological, and motor skills that help them achieve the objectives proposed at each school level, since their learning is determined by an efficient management of their potential from an integral perspective. However, when the didactic action moves away from these precepts it adds to deterioration in the educational quality, it is there, where 
Análisis de la actividad física y su importancia en el sistema educativo ecuatoriano: carrera Gestión Emresarial de la Universidad Estatal de Sur de Manabí

the Law of Education and Culture (1983) in its article 2 numeral 1 - 2 that "it is a primary duty of The State to that all Ecuadorians have a comprehensive education and the obligation to participate actively in the national educational process. " (p.1) Therefore, the students of the business management career, have opportunities to use their physical, motor, social, personal development during their academic activities, resize their human condition and build the bases to adapt their professional tasks in relation to the job responsibilities as manager or administrator that precedes their performance; Being prepared under a physical activity, they develop as individuals with possibilities to discover and cultivate the qualities that are going to form it integrally.

Palavras chaves: Atividade física; Sistema Educacional Equatoriano; Carreira de Gestão Empresarial; Universidade Estadual do Sul Manabí.

\section{Introducción.}

La educación a lo largo de la historia se ha convertido en la herramienta fundamental para garantizar a los ciudadanos y ciudadanas una formación integral con oportunidades comunes que le ayuden a incursionar en el campo de trabajo como resultado a la adquisición de destrezas y habilidades profesionales fomentadas durante el cumplimiento de toda una gama de tareas encargadas de ofrecerles situaciones para descubrir sus propias potencialidades. Ante este reto permanente cada nación desarrolla políticas educativas dirigidas a mejorar el sistema educativo con el fin de abarcada un mayor número de individuos y afianzar con ello un progresivo progreso en las actividades económicas que darán como resultado la presencia de una sociedad determinada por un crecimiento integral.

En este sentido, Santos (2015) precisa que el área prioritaria de la política pública "lo representa la inversión estatal que garantiza igualdad e inclusión social y condiciones indispensables para el buen vivir, mediante una educación de calidad". (p.51). Por lo tanto, para hablar de cambios integrales en la vida social, se hace necesario entender la significación que posee la educación como 
Juan M. González-Morán; Marcos G. Cañarte-Castillo; Rubén D. Acebo-Calderón; Regalado F. Katty-Glenda; Carlos A. Zea-Barahona; Xavier E. Soledispa-Rodríguez

pilar determinante para mejorar los diferentes contextos que estructuran a una nación. Es decir, formar a los individuos es asignarle oportunidades comunes que dan respuestas oportunas a sus necesidades y aseguran con anticipación el buen vivir como representación de su formación profesional.

Al vincular estas apreciaciones con el sistema educativo ecuatoriano, se puede indicar que el mismo ha creado sistemas políticos, económicos, educativos, que permitan obtener este fin último de la sociedad, todo ello ajustado a las realidades y evolución del sistema debidamente compaginado con los aportes internacionales, es decir, dar apertura a estas diferentes formas que dieron mayor o menor éxito. En consecuencia, la educación ecuatoriana se identifica como una actividad global encargada de agilizar el motor del desarrollo, pues asegura con su dinámica la formación integral de los recursos humanos y la capacidad de producir bienes y servicios que satisfagan las necesidades básicas de la sociedad.

En consecuencia, mejorar la calidad educativa en Ecuador se convierte en un tema discutido en los últimos años, cuya finalidad es establecer una propuesta que logre en la práctica una verdadera reforma curricular para consolidar las políticas de Estado. Por lo tanto, los especialistas en educación coinciden en que la única fórmula para salir del subdesarrollo es a través de un nuevo modelo educativo, que apunte hacia una alternativa de carácter global a los problemas de la sociedad, que proponga como elemento esencial una reforma de este proceso promovido por los propios educadores y con la participación de la comunidad.

De este modo, se entiende que el Ecuador como nación para convertir a la educación en un modelo centrado en la competitividad, busca mediante reformas diseñadas como una estrategia para alcanzar la calidad desde un punto de vista administrativo, donde se evidencie el respectivo vínculo 
Análisis de la actividad física y su importancia en el sistema educativo ecuatoriano: carrera Gestión Emresarial de la Universidad Estatal de Sur de Manabí

estrecho entre la calidad educativa y los recursos que el Estado asigna para alcanzar dicho propósito. Al respecto, Baldallo (2017) destaca que "la educación constituye uno de los ejes estratégicos para el desarrollo nacional, pues, la misma tiene la responsabilidad de formar las nuevas generaciones”. (p.36)

Al tomar en consideración este planteamiento, se puede indicar que las universidades dentro del sistema educativo ecuatoriano, deben concebir la acción didáctica desde un contexto estratégico que haga posible ayudar a los estudiantes a la adquisición de habilidades con el fin de responder al desarrollo de grandes procesos formativos en los ámbitos de las competencias profesionales, conocimiento, habilidades, destrezas, capacidades, aptitudes, actitudes y valores, desempeño idóneo, integral de conformidad a las exigencias necesarias, dinámicas y transformaciones sociales vigentes en el Ecuador.

En el marco de estas ideas, se puede complementar que, las universidades como instituciones encargadas de formar aquellos profesionales en las diferentes áreas del conocimiento, deben reconocer con anticipación las competencias presente en los grupos, con el fin de garantizar su desarrollo personal y social, ajustado a las necesidades políticas, económicas, culturales y educativas que vive la nación. Es decir, debe concebir aquellas habilidades psicofísicas que trae el estudiante para afianzar durante la ejecución de su práctica académica, nuevas situaciones que al ser combinadas con las informaciones previas, consolide en ellos destrezas profesionales en función de las competencias particulares preestablecidas en cada carrera a dictar.

De este modo, el Estado ecuatoriano en búsqueda de nuevos cambios propiciadores de una acción dinámica que introduzca transformaciones en la vida escolar, introduce la actividad física 
Juan M. González-Morán; Marcos G. Cañarte-Castillo; Rubén D. Acebo-Calderón; Regalado F. Katty-Glenda; Carlos A. Zea-Barahona; Xavier E. Soledispa-Rodríguez

como un elemento esencial en los niveles del sistema educativo, para lo cual, el Ministro de Educación (2018) indica con el fin de fortalecer la "actividad física a través de suficientes alternativas para que el país vaya supliendo en corto plazo el déficit de profesores en Educación Física”.(p.3) Estas ideas, precisan la necesidad de gestionar cambios puntuales en la formación integral de los estudiantes, es decir, el Estado concibe la acción educativa como una respuesta viable que desarrolle en los estudiantes su formación integral, por ello, visualizan la actividad física como una alternativa competente e innovadora que les ayuda a consolidar su desarrollo personal desde un criterio integral.

Este mismo organismo acota "se va a emprender un plan de capacitación mediante convenio con docentes de universidades ecuatorianas; además de la presencia de maestros que se van a incorporar en este año a la actividad física”. (p.3). Es decir, las instituciones universitarias tanto públicas como privadas, poseen una corresponsabilidad en cuanto al desarrollo de este objetivo centrado en introducir la actividad física como parte esencial del sistema educativo, este aporte, se convierte en un factor esencial para garantizar no sólo en los estudiantes de otros niveles educativos, sino, proyectar en las diferentes carreras establecidas una evaluación esencial que le ofrezca dimensiones esenciales para reconocer las habilidades, destrezas, competencias y aptitudes que poseen los estudiantes y así redimensionar su formación integral para obtener en el campo laboral la presencia de profesionales competentes y eficientes.

De igual manera, se puede indicar lo indicado por esta institución pública en cuanto a la actividad física significa "educar a través del movimiento, es decir acciones que impliquen un desplazamiento del cuerpo o de segmentos corporales, e implica la mente que dirige los movimientos conscientes hacia un objetivo".(p.4) Según la apreciación, se entiende que la actividad física en el marco del sistema educativo ecuatoriano, no es vista como una práctica competitiva que 
Análisis de la actividad física y su importancia en el sistema educativo ecuatoriano: carrera Gestión Emresarial de la Universidad Estatal de Sur de Manabí

ayude a los estudiantes no sólo a generar movimientos de sus segmentos corporales, sino lograr el desarrollo de la mente como parte esencial para garantizar en los estudiantes actitudes que le faciliten la toma de decisiones en un momento determinado.

No obstante, Castaño (2017) precisa que "uno de los problemas que tiene la inclusión de las actividades físicas en el sistema educativo, se centra en que se habla de deporte y se crean muchas políticas deportivas y práctica física, pero no es vista como educación”.(p.11). Este argumento, permite reflexionar en cuanto a la importancia que posee la actividad física en el sistema educativo, pues, el autor valorar una realidad distante en dicha materia, donde el Estado ecuatoriano carece de una visión integral que conciba a la actividad física como un conglomerado de principios básicos y epistemológicos que garantice a todos los niveles del sistema educativo la trayectoria de la práctica física como agente de cambio en la vida de los estudiantes.

Cada una de las consideraciones citadas anteriormente, llevan a precisar que el sistema educativo ecuatoriano, debe concebir la actividad física desde una visión educativa, que le ayude a cada estudiante a crear una cultura por el gusto de la actividad física, donde sea guiado y autónoma que le facilite condiciones saludables por encima de la estética, pues ésta se convierte en la primera base de dicha práctica. Tal como lo asevera Ceballos (2019) "el estudiante ecuatoriano al cumplir actividades físicas corporales se encuentra íntimamente relacionado con las prácticas mentales de control del movimiento y concentración”. (p.22). Por lo tanto, cuando los estudiantes universitarios hacen la selección de su carrera profesional, las instituciones superiores complementan su carga académica con dichos procesos corporales y mentales adquiridos a lo largo de su formación 
Juan M. González-Morán; Marcos G. Cañarte-Castillo; Rubén D. Acebo-Calderón; Regalado F. Katty-Glenda; Carlos A. Zea-Barahona; Xavier E. Soledispa-Rodríguez

educativa, para así finalmente lograr su formación plena y ofrecerle a la sociedad profesionales altamente calificados.

Según los planteamientos citados a lo largo del discurso, se estima que la actividad física ejerce una importancia significativa dentro del sistema educativo ecuatoriano, mediante su desarrollo se hace posible cumplir con una práctica social en la que resalta el éxito, esfuerzo y la aceptación de errores cuando sucedes al momento de compartir espacios y actividades con otras personas. Además, hace posible difundir informaciones relativas a la alimentación, nutrición consciente, pues, se conoce que la mejor medicina para el ser humano es la actividad física y una correcta alimentación, Para Ceballos (ob.cit) cuando los estudiantes se encuentran alejados de la actividad física "están propensos a enfermedades que amenazan la integridad como hipertensión e infartos" (p.26)

De este modo, se puede indicar que los estudiantes que actualmente cursan la carrera de gestión empresarial en la Universidad Estatal del Sur de Manabí, por estar incursos durante sus estudios en los diferentes niveles del sistema educativo bajo el desarrollo de la actividad física, logran llegar a la universidad con competencias personales, destrezas motoras que le ayudan a mejorar sus capacidad de atención y concentración durante el desarrollo del proceso educativo; además, amplían sus habilidades emocionales que le facilitan el reconocimiento de las dificultades y tomar decisiones oportunas para así resolver sus posibles conflictos sociales y académicos de manera precisa a la situación presentada en sus relaciones sociales vividas en el ambiente universitario.

También se puede decir, que la existencia de la actividad física en el sistema educativo ecuatoriano, al ser visualizado con este grupo de estudiantes les ayuda a elevar sus capacidades para 
Análisis de la actividad física y su importancia en el sistema educativo ecuatoriano: carrera Gestión Emresarial de la Universidad Estatal de Sur de Manabí

emprender proyectos, trabajar grupal e individualmente, alcanzar una formación profesional con ética que le ayuda a encontrar la armonía individual como conciencia ecológica y social; asimismo, le asegura el éxito al momento de aplicar las estrategias en los procesos empresariales; además logra, registrar análisis de los problemas económicos, sociales, financieros de las empresas para facilitar la construcción de nuevas visiones que ayuden a fortalecer los objetivos trazados. Tal como lo asevera Grumer (2015) la actividad física en los estudiantes “es una forma de intervención que construye y reconstruye identidades sociales y posiciones relativas del poder" (.71)

De acuerdo con los planteamientos dados se precisa, que el sistema educativo ecuatoriano ha buscado a lo largo de su desarrollo la implementación de nuevas acciones centradas en la actividad física como una forma específica que ayude a los estudiantes en sus diferentes niveles a consolidar las destrezas motoras, personales, sociales y cognitivas que sirven de referencia para convertirlos en autores de sus propias decisiones y obtener el éxito durante la su formación profesional, de esta manera, las universidades responden positivamente ante dicha realidad al concebir sus estructuras organizativas desde una criterio de identificación de las competencias presente en cada grupo.

Los aportes dados a lo largo de este discurso, se puede indicar que los mismo sirven de referencia para el desarrollo de este artículo, mediante el cual se llega a un análisis de la actividad física y su importancia en el sistema educativo ecuatoriano: carrera gestión empresarial de la Universidad Estatal del Sur de Manabí, a través de sus valoraciones se aportan relevantes encargados de proyectar sus consideraciones en un campo científico particular.

\section{Método.}

El accionar investigativo requiere aplicar estrategias idóneas que hagan posible encaminar el respectivo recorrido preestablecido desde una dinámica innovadora, por ello, se introduce la 
Juan M. González-Morán; Marcos G. Cañarte-Castillo; Rubén D. Acebo-Calderón; Regalado F. Katty-Glenda; Carlos A. Zea-Barahona; Xavier E. Soledispa-Rodríguez

conceptualización dada por Escalona (2017) “el proceso investigativo necesita el apoyo de un método para responder a su propósito de forma dinámica y ajustado a las necesidades previamente encontradas”.(p.48) Es decir, para encaminar el trabajo en curso es importante acoplar su contenido bajo la dirección de un método relacionado con los fines de la investigación.

Según lo planteado, muestra claramente que el trabajo científico para garantizar su desarrollo en forma armónica a los principios establecidos, debe ser encaminado mediante la presencia de un método acoplado a la necesidad del hecho por indagar. Es así como, el presente artículo recoge diferentes actuaciones de los contenidos propuestos para finalmente fijar una posición correspondiente al interés de los investigadores.

\section{Tipo de Investigación}

El desarrollo del contenido previamente seleccionado más su propósito llevan a ubicarlo dentro de un tipo de investigación identificada como documental, apoyada en la definición dada por Escalona (ob.cit) "representa un trabajo centrado en la recopilación de informaciones obtenidas de materiales bibliográficos y tecnológicos que están directamente vinculados con el tema seleccionado".(p.50). Por lo tanto, para darle continuidad al trabajo se tuvo que acudir a la revisión, selección y lectura previa de diferentes documentos legales, libros y diferentes materiales impresos para así asignar los respectivos aportes.

Asimismo, se puede indicar que para darle flexibilidad a este proceso de indagación se incorporó el diseño bibliográfico visto por el autor citado "como un medio encargado de llevar al investigador a recopilar informaciones básicas que darán respuestas a los fines trazados en el hecho investigativo". (p.50). Es decir, mediante este aporte se dará cabida a un proceso científico, donde 
Análisis de la actividad física y su importancia en el sistema educativo ecuatoriano: carrera Gestión Emresarial de la Universidad Estatal de Sur de Manabí

cada investigador, tuvo la oportunidad de seleccionar sus aportes en estrecha relación con las características del fenómeno en estudio.

\section{Fuentes Documentales}

Toda investigación para lograr adecuar sus espacios con eficiencia, debe estar encaminada bajo la mirada de fuentes documentales básicas que le permitan asegura el desarrollo de cada uno de los planteamientos considerados importantes en el marco del hecho investigativo, es así como, Figuera (2018) define a las fuentes documentales "como la información de origen que le ofrece al investigador literaturas diferentes para cumplir con los fines propuestos en un momento determinado". (p.23) Este planteamiento, permite, entender que el apoyo de las fuentes documentales, le permita al investigador seleccionar el material encargado de dar la operatividad al proceso investigativo, pues, mediante la utilización de diferentes tareas logra abrir el camino para proporcionar nuevas informaciones funcionales que abren nuevas formas de interpretar los hechos que fueron debidamente acoplados al interés de los encargados de estudiar el tema seleccionado.

\section{Técnicas de Recolección de la Información}

Los aspectos relativos a este tópico, representa un momento especial, pues, asume la selección previa de aquellas herramientas básicas para leer e interpretar los contenidos requeridos en el proceso investigativo, es así, se emplearon como técnica el fichaje definido por, Escalona (ob.cit), “como una actividad de revisión y selección de documentos que permiten explicar tópicos específicos" (p.47). En otras palabras, los investigadores tuvieron la oportunidad de seleccionar los tópicos necesarios para llevar a cabo el proceso investigativo. 
Juan M. González-Morán; Marcos G. Cañarte-Castillo; Rubén D. Acebo-Calderón; Regalado F. Katty-Glenda; Carlos A. Zea-Barahona; Xavier E. Soledispa-Rodríguez

En esta misma dirección, se hizo necesario la introducción de la técnica del resumen, medio mediante el cual, se logra cumplir la fase correspondiente a la interpretación para luego extraer nuevas ideas, tal como lo define Figuera (ob,.cit), “consiste en la interpretación de contenidos en forma analítica"(p.66). Es decir, una vez seleccionada las diferentes fuentes teóricas, se procedió a su organización en forma coherente a los criterios científicos.

Dentro de todo proceso investigativo, corresponde estimar una gama de procedimientos o momentos básicos que dan cabida al desarrollo de los elementos conformadores del tema en estudio. Tal como lo indica Figuera (ob.cit), "conjunto de acciones progresivas que permiten el perfeccionamiento de un estudio, para plasmar posibles resultados efectivos al análisis” (p.93). En consecuencia, la incorporación de las diferentes técnicas dieron como resultado, la realización de un revisión de documentos: Caracterizada como la fase previa a la formulación del problema, mediante la cual se consideraron los aportes esenciales para dar continuidad al proceso investigativo, centrado en el manejo de diversas fuentes documentales encargadas de darle la respectiva claridad y coherencia a los tópicos a desarrollar en el contenido. Además, se cumplió el respectivo apoyo bibliográfico, para lograr el respectivo ajuste de las fuentes documentales encargadas de ofrecer informaciones básicas y especiales que hicieron posible el respectivo engranaje de los eventos propuestos, es decir, construir cada uno de los eventos que estructuran el artículo en curso.

\section{Resultados.}

Actividad Física

Existe una concepción generalizada de la relación directa entre la práctica de la actividad física y los efectos beneficiosos que proporciona para la salud. Desde diversos sectores como: medicina, fisioterapia, psicología y educación física, se destaca el papel esencial que el correcto 
Análisis de la actividad física y su importancia en el sistema educativo ecuatoriano: carrera Gestión Emresarial de la Universidad Estatal de Sur de Manabí

ejercicio físico posee desde el punto de vista preventivo en la mayoría de las alteraciones que se producen con frecuencia en la sociedad. Los medios de comunicación se han encargado de explotar este valor en alza como producto de consumo y, de esta forma, todos los sectores sociales están informados sobre la necesidad de incluir el ejercicio físico como hábito para conseguir una vida sana y una óptima calidad de vida

Por ello, desde tiempos inmemoriales el ser humano ha vinculado la práctica de la actividad física al buen estado corporal y la salud, en relación a los tiempos modernos se plantea como algo más que una lucha contra la enfermedad, en consecuencia, cada país al momento de estructurar su modelo educativo busca no sólo orientarlo bajo la perspectiva de la actividad física, sino que fija posiciones claras en cuanto a la necesidad de mejorar progresivamente la salud de los individuos. Al respecto, Martí (2015) destaca la calidad de vida "se encuentra determinada por la relación que hace el sujeto con el medio ambiente y la cultura". (p.16)

En consecuencia, cuando la actividad física representa para la población ecuatoriana una mágica experiencia competente que le dará cabida a responder a sus necesidades desde un contexto particular. Es decir, se introduce el concepto desde la concepción de salud, visto por Valera (2015) “el completo estado de bienestar físico, psíquico y social”.(p.22). De este modo, al incluir en las mallas curriculares la presencia de la actividad física, se precisa su importancia en los estudiantes universitarios, pues, da apertura a lograr no sólo la consolidación de un cuerpo sano, sino que propicia un ambiente emocional saludable que hace posible direccionar sus actividades académicas bajo una acción controlada y mejorada progresivamente para alcanzar el éxito.

En esta misma dirección la Organización Mundial de la Salud (2015) introduce un nuevo concepto en cuanto al actividad física, "vista como aquella acción insertada con el medio social 
Juan M. González-Morán; Marcos G. Cañarte-Castillo; Rubén D. Acebo-Calderón; Regalado F. Katty-Glenda; Carlos A. Zea-Barahona; Xavier E. Soledispa-Rodríguez

donde se desenvuelve el sujeto y atendido por una persona como individualidad".(p. 18). Este nuevo criterio, permite vincular la actividad física en el campo educativo, como una forma direccionada bajo la presencia del docente quien responderá de forma individual ante cada estudiante, tomando como principio básico el entorno circundante para responder a sus propias necesidades y lograr el respectivo desarrollo integral que requiere para acoplarse al medio social.

Bajo esta perspectiva, es de destacar que las definiciones anteriores, permite entender que el criterio epistemológico que caracteriza a la actividad física, lleva a valorar el medio social donde el sujeto se circunscribe, además, la necesidad de un individuo que propicie las condiciones reales para adecuar los espacios básicos que le dará apertura a un nuevo individuo caracterizado por la presencia de controles emocionales, manejo efectivo de su cuerpo, entendimiento de las reglas o normas instruccionales como medidas para alcanzar respuestas oportunas en un ámbito educativo. Dado que, se engloban factores multidimensionales caracterizados por considerar los aspectos sociales, físicos, intelectuales, espirituales e intelectuales que estructuran la personalidad de los estudiantes.

Al valorar las apreciaciones citadas en párrafos anteriores, se puede indicar que la actividad física, debe tener como note en el campo educativo, la presencia del dinamismo determinado por el desarrollo de la interdisciplinariedad, pues, todos los agentes sociales e institucionales tienen responsabilidades en su promoción. En otras palabras, cada organización educativa que ocupa un espacio en el sistema educativo ecuatoriano, no puede dejar a un lado la respectiva valoración de la actividad física, es importante reconocer su oportuna intervención en la vida humana, pues, encamina al estudiante hacia la programación y secuenciación de los contenidos que recoge el área de educación física. 
Análisis de la actividad física y su importancia en el sistema educativo ecuatoriano: carrera Gestión Emresarial de la Universidad Estatal de Sur de Manabí

Desde esta visión, se comprende que el currículo construido para lograr un adecuado desarrollo de las actividades físicas en los planteles educativos, es hacer manejar una didáctica explicita que oriente a los docentes hacia el interés de descubrir en sus estudiantes las potencialidades en cuanto a su condición física, género, aptitudes, manejo del cuerpo, movimientos, expresión corporal, atención, concentración, percepción, cada uno de estos elementos son los encargados de precisar su importancia en el campo educativo, en tal sentido, Evans (2018) destaca “el proceso educativo para fomentar la actividad física debe ser concebido para enseñar a los estudiantes a ser activos, autónomos de por vida e independientes”.(p.47)

Estas consideraciones, llevan a comprender que en la medida que los estudiantes tengan una actividad física debidamente programada, logran resultados significativos en el desarrollo del pensamiento interior, pueden reducir la presencia de enfermedades, generan altas posibilidades para aprender con mayor facilidad debido al incremento de su atención y concentración, además promociona en ellos estados emocionales positivos que aseguran el éxito durante el desarrollo de sus actividades académicas; asimismo, intervienen abiertamente en momentos comunicacionales con sus pares para garantizar con ello beneficios colectivos e individuales. Para Blair (2016) la actividad física "es una respuesta beneficiosa al trabajo en general, en el campo educativo, asegura la suma de diferentes momentos saludables que le permiten al estudiante mejorar sus competencias en general”. (p.59)

Cabe agregar que al traspolar estas informaciones hacia los estudiantes de gerencia empresarial, se puede entender la significación que posee para ellos, el ser formados en un contexto donde la actividad física sea el pilar educativo, pues, los mismos adquieren destrezas psicosociales que le ayudan a mantener contactos empáticos y garantizan con ello, la consolidación de sus capacidades para convertirse en líderes al momento de cumplir sus actividades administrativas 
Juan M. González-Morán; Marcos G. Cañarte-Castillo; Rubén D. Acebo-Calderón; Regalado F. Katty-Glenda; Carlos A. Zea-Barahona; Xavier E. Soledispa-Rodríguez

concernientes a sus funciones durante el ejercicio profesional que le compete. Es decir, que mediante estas acciones dirigidas a que reconozca su cuerpo como un medio de conocimiento que le dará apertura a una toma de conciencia de su corporedad y comunicarse a través del mismo al momento de ejecutar su desempeño profesional.

\section{Sistema Educativo Ecuatoriano}

La educación escolarizada es acumulativa, progresiva y conlleva la obtención de un título o certificado. Esta puede ser catalogada en ordinaria o extraordinaria (Reglamento General a la LOEI, 2011: Art. 23). Mientras la oferta ordinaria atiende a estudiantes en los rangos de edades preferentes, la oferta extraordinaria atiende mayoritariamente a personas con escolaridad inconclusa de 15 años o más, que han sobrepasado la edad correspondiente y requieren otras modalidades para la culminación de sus estudios. Algunos indicadores pueden reportar estudiantes de menor edad, debido a que en ciertos lugares este tipo de oferta educativa es la única.

Mientras que la educación no escolarizada provee espacios educativos a lo largo de la vida y su currículo se adapta a las necesidades o particularidades del sector de la población que atiende, caracterizándose como una oferta educativa esporádica y con una duración menor a un año escolar. Este tipo de educación implica cursos temporales que no conducen a la obtención de un título o certificado como la escolarizada.

En cuanto a la organización del sistema educativo ecuatoriano, se puede indicar que se encuentra conformado por niveles los cuales se destacan a continuación: 
Análisis de la actividad física y su importancia en el sistema educativo ecuatoriano: carrera Gestión Emresarial de la Universidad Estatal de Sur de Manabí

Educación Inicial: Es optativo, no es requisito indispensable para que el estudiante pueda iniciar su Educación General Básica. Es un nivel reciente, aún no ha sido implementado en todos los planteles educativos. A su vez comprende:

Inicial 1: No escolarizado, para niños de hasta 3 años de edad. El responsable de la regulación de los servicios de Desarrollo Infantil Integral (DII), correspondientes a esta etapa, es el MIES. En este nivel se integra a los padres en la educación no formal de sus hijos a través de actividades lúdicas educativas. Los centros organizan sus propias jornadas de acuerdo a la realidad de la ciudad en la que se encuentran ubicados. La jornada (Mañana o tarde) también es establecida por cada centro. El objetivo de este nivel es estimular el desarrollo integral de los niños y, su prioridad, fomentar la inclusión y el respeto por la diversidad.

En cuanto a la inscripción se puede decir que el proceso es sencillo, el padre de familia acude al o los centros del sector en el que vive con un comprobante de servicio básico y la partida de nacimiento o cédula del niño. Con estos documentos el centro asigna los cupos. En caso de que las solicitudes excedan la disponibilidad del centro, se asignarán los cupos mediante sorteo público. Los niños que no salen favorecidos con un cupo serán ubicados en centros que dispongan de cupos o, en última instancia serán solicitados cupos adicionales.

Inicial 2: Para niños de 3 hasta 5 años. Desde esta etapa la educación de los niños pasa a ser atendida por el Ministerio de Educación. Se incorporan actividades como: la toma de asistencia, trabajar el calendario, estimulación del lenguaje oral, dialogar noticias o eventos especiales. Se busca que los niños se expresen con libertad.

Para cumplir con la inscripción en este nivel deben ser matriculados los niños de entre 3 y 4 años, para que a su salida puedan cumplir con el requisito de Ley (LOEI) de tener al menos 5 años 
Juan M. González-Morán; Marcos G. Cañarte-Castillo; Rubén D. Acebo-Calderón; Regalado F. Katty-Glenda; Carlos A. Zea-Barahona; Xavier E. Soledispa-Rodríguez

de edad para poder ingresar al Nivel de Educación General Básica. Para la matrícula deben presentar original y copia de la partida de nacimiento o cédula de ciudadanía (no de ambas) y un certificado de salud.

Educación General Básica: Comprende: en el Ecuador abarca desde primer hasta décimo grado, a través de los cuales los estudiantes adquieren un conjunto de capacidades y responsabilidades a partir de tres valores fundamentales que forman parte del perfil del bachiller ecuatoriano: la justicia, la innovación y la solidaridad. Los estudiantes que terminan este nivel, serán capaces de continuar los estudios de Bachillerato y participar en la vida política y social, conscientes de su rol histórico como ciudadanos ecuatorianos. También, abarca la Preparatoria (Primero de básica): Alumnos de 5 años. Materias que revisan: Entorno natural y Social, educación estética y educación física. Básica Elemental (2do, 3ro y 4to de básica) De 6 a 8 años. Asimismo, se encuentra la Básica media (5to, 6to y 7mo de básica) de 9 a 11 años.

Básica Superior: (8vo, 9no y 10mo de básica) De 12 a 14 años. Materias que revisan: 8vo, 9no y 10mo: Lengua y literatura, matemática, ciencias naturales, estudios sociales, educación estética, educación física, clubes y lengua extranjera.

Bachillerato General Unificado: Es el último nivel de educación obligatoria. Este nivel es nuevo, fue diseñado con el objetivo de preparar mejor a los bachilleres, la primera promoción graduada recién fue recibida por las universidades en el segundo semestre de 2014. Tiene una duración de 3 años y lo cursan los estudiantes desde los 15 a 17 años de edad. Aprobado se obtiene el Título de Bachiller. Sobre las asignaturas que cursan, hay una base común de materias llamada Tronco común que deben cursar todos. Luego, los estudiantes optan por: Bachillerato en Ciencias o bachillerato Técnico. 
Análisis de la actividad física y su importancia en el sistema educativo ecuatoriano: carrera Gestión Emresarial de la Universidad Estatal de Sur de Manabí

Para el proceso de inscripción en estos niveles se hace necesario la (Fase inscribe): En un período de tiempo y en sedes dispuestas por el Ministerio de Educación se realiza la inscripción de los alumnos. Si por algún motivo no alcanzó a inscribir a su hijo, posteriormente dan un tiempo para inscripciones tardías. El Ministerio dará cupos a todos los estudiantes que se inscriban

Concluido el bachillerato, los estudiantes pasan a cursar sus estudios superiores universitarios y el ente Rector pasa a ser la Senescyt.

Es importante acotar que el sistema educativo ecuatoriano, se encuentra caracterizado por una misión que busca garantizar el acceso y calidad de la educación inicial, básica y bachillerato a los y las habitantes del territorio nacional, mediante la formación integral, holística e inclusiva de niños, niñas, jóvenes y adultos, tomando en cuenta la interculturalidad, la plurinacionalidad, las lenguas ancestrales y género desde un enfoque de derechos y deberes para fortalecer el desarrollo social, económico y cultural, el ejercicio de la ciudadanía y la unidad en la diversidad de la sociedad ecuatoriana.

Al hacer referencia a la visión el Sistema Nacional de Educación brindará una educación centrada en el ser humano, con calidad, calidez, integral, holística, crítica, participativa, democrática, inclusiva e interactiva, con equidad de género, basado en la sabiduría ancestral, plurinacionalidad, con identidad y pertinencia cultural que satisface las necesidades de aprendizaje individual y social, que contribuye a fortalecer la identidad cultural, la construcción de ciudadanía, y que articule los diferentes niveles y modalidades del sistema de educación.

Por lo tanto, para alcanzar la misión y visión se traza una serie de valores fundamentales entre los cuales se citan los siguientes: Honestidad, para tener comportamientos transparentes - 
Juan M. González-Morán; Marcos G. Cañarte-Castillo; Rubén D. Acebo-Calderón; Regalado F. Katty-Glenda; Carlos A. Zea-Barahona; Xavier E. Soledispa-Rodríguez

honradez, sinceridad, autenticidad, integridad- con nuestros semejantes y permitir que la confianza colectiva se transforme en una fuerza de gran valor.

Justicia, para reconocer y fomentar las buenas acciones y causas, condenar aquellos comportamientos que hacen daño a los individuos y a la sociedad, y velar por la justicia a fin de que no se produzcan actos de corrupción. Respeto, empezando por el que nos debemos a nosotros mismos y a nuestros semejantes, al ambiente, a los seres vivos y a la naturaleza, sin olvidar las leyes, normas sociales y la memoria de nuestros antepasados.

Paz, para fomentar la confianza en nuestras relaciones con los demás, para reaccionar con calma, firmeza y serenidad frente a las agresiones, y para reconocer la dignidad y los derechos de las personas. Solidaridad, para que los ciudadanos y ciudadanas colaboren mutuamente frente a problemas o necesidades y se consiga así un fin común, con entusiasmo, firmeza, lealtad, generosidad y fraternidad.

Responsabilidad, para darnos cuenta de las consecuencias que tiene todo lo que hacemos o dejamos de hacer, sobre nosotros mismos o sobre los demás, y como garantía de los compromisos adquiridos. Pluralismo, para fomentar el respeto a la libertad de opinión y de expresión del pensamiento, y para desarrollar libremente personalidad, doctrina e ideología, con respeto al orden jurídico y a los derechos de los demás.

\section{CONCLUSIONES.}

El desarrollo de los diversos contenidos presentados a lo largo del artículo, permiten llegar a precisar una serie de apreciaciones generales entre las cuales se puede indicar lo siguiente: Es importante indicar que mediante la realización de la actividad física los individuos logran tener una 
Análisis de la actividad física y su importancia en el sistema educativo ecuatoriano: carrera Gestión Emresarial de la Universidad Estatal de Sur de Manabí

adecuada condición física cardiorrespiratoria que refleja la presencia de niveles óptimos de salud que le permiten a cada individuo tener confianza en sí mismo y buscar alternativas viables para resolver los problemas presente en su vida diaria.

De este modo, se puede decir que la actividad física debe ser vista como una forma de agilizar el crecimiento y desarrollo de diferentes destrezas competitivas que facilitan en los estudiantes oportunidades comunes, al momento de establecer interrelaciones con la práctica escolar. Mediante el desarrollo de esta acción se logra reforzar beneficios considerables para salud del individuo que la cumple. En el caso de los estudiantes universitarios de la carrera gestión empresarial, se puede deducir que los mismos, logran encontrar durante el ejercicio profesional cambios progresivos en sus tareas financieras y administrativas.

Por lo tanto, adecuar la acción pedagógica bajo la dirección de la actividad física lleva a encontrar una significativa importancia, dado que aporta a la acción escolar un cambio progresivo al encontrar que los estudiantes son debidamente canalizados en su desarrollo psicológico, social, motor e intelectual. Eventos que dan cabida a la organización de situaciones vivenciales para así consolidar en ellos aptitudes o competencias básicas que le servirán de base al momento de incursionar en el nivel de educación superior, donde requieren incorporar sus habilidades cognitivas para resolver problemas desde sus propias competencias personales.

Otro aspecto de relevancia, lo constituye la estructuración del sistema educativo ecuatoriano, que se estima en niveles que parten desde el inicial, aunque no reúne características de obligatorio busca ofrecer a los niños y niñas oportunidades comunes en su formación social y emocional. Mientras que la básica elemental hace un recorrido por la educación primaria hasta alcanzar la 
Juan M. González-Morán; Marcos G. Cañarte-Castillo; Rubén D. Acebo-Calderón; Regalado F. Katty-Glenda; Carlos A. Zea-Barahona; Xavier E. Soledispa-Rodríguez

formación completa que llega al bachillerato y de allí puede simplemente ser inscrito en las instituciones públicas o privadas que cumplen la visión y misión de la educación superior.

Finalmente se puede indicar que la actividad física en el campo educativo fomenta en los estudiantes influyentes condiciones ambientales para lograr en los estudiantes comportamientos efectivos que los llevan a aprender de forma significativa, razón por la cual, es vista como una herramienta funcional dentro del sistema educativo ecuatoriano, el Estado a través de ella garantiza a todos los estudiantes en sus diferentes niveles oportunidades de interconectarse con nuevas vivencias para fomentar entre ellos competencias socializadoras que abren el camino motivacional hasta alcanzar el éxito profesional.

\section{Referencias Bibliográficas}

Baldallo, T. (2017). Educación: Compleja Visión Humana . México: Mesa Redonda .

Blair, D. (2016). Salud y Desarrollo . México: Educativo .

Castaño, P. (2017). Políticas Educativas en Ecuador. México : Isis .

Ceballos, D. (2019). Aptitudes y Práctica Física . Madrid : Morata .

Educación, M. d. (2018). Educación Ecuatoriana .

Escalona, H. (2017). Investigación Documental . Caracas : Panapo .

Evans, E. (2018). Actividad Física . México : Urania .

Figuera, L. (2018). Técnicas Bibliográficas . Barcelona : Gedisa .

Grumer, R. (2015). Actividad Física . Chile : Paidós .

Martí, B. (2015). Vivir Saludable . España : Narcea .

Salud, O. M. (2015). Visión Retrospectiva de la Salud.

Santos, I. (2015). Calidad Educativa . Caracas : Oasis .

Valera, T. (2015). Educación Deportiva . Caracas : Greco. 